\title{
Evaporating Spray in Supersonic Streams Including Turbulence Effects
}

\author{
M. S. Balasubramanyam ${ }^{*}$, C. P. Chen ${ }^{\ddagger}$ \\ University of Alabama in Huntsville, Huntsville, AL 35899 \\ and \\ H. P. Trinh ${ }^{\S}$ \\ NASA-Marshall Space Flight Center, Huntsville, AL 35812
}

Evaporating spray plays an important role in spray combustion processes. This paper describes the development of a new finite-conductivity evaporation model, based on the two-temperature film theory, for two-phase numerical simulation using Eulerian-Lagrangian method. The model is a natural extension of the T-blob/T-TAB atomization/spray model $^{1}$ which supplies the turbulence characteristics for estimating effective thermal diffusivity within the droplet phase. Both one-way and two-way coupled calculations were performed to investigate the performance of this model. Validation results indicate the superiority of the finite-conductivity model in low speed parallel flow evaporating sprays. High speed cross flow spray results indicate the effectiveness of the T-blob/T-TAB model and point to the needed improvements in high speed evaporating spray modeling.

\section{Nomenclature}

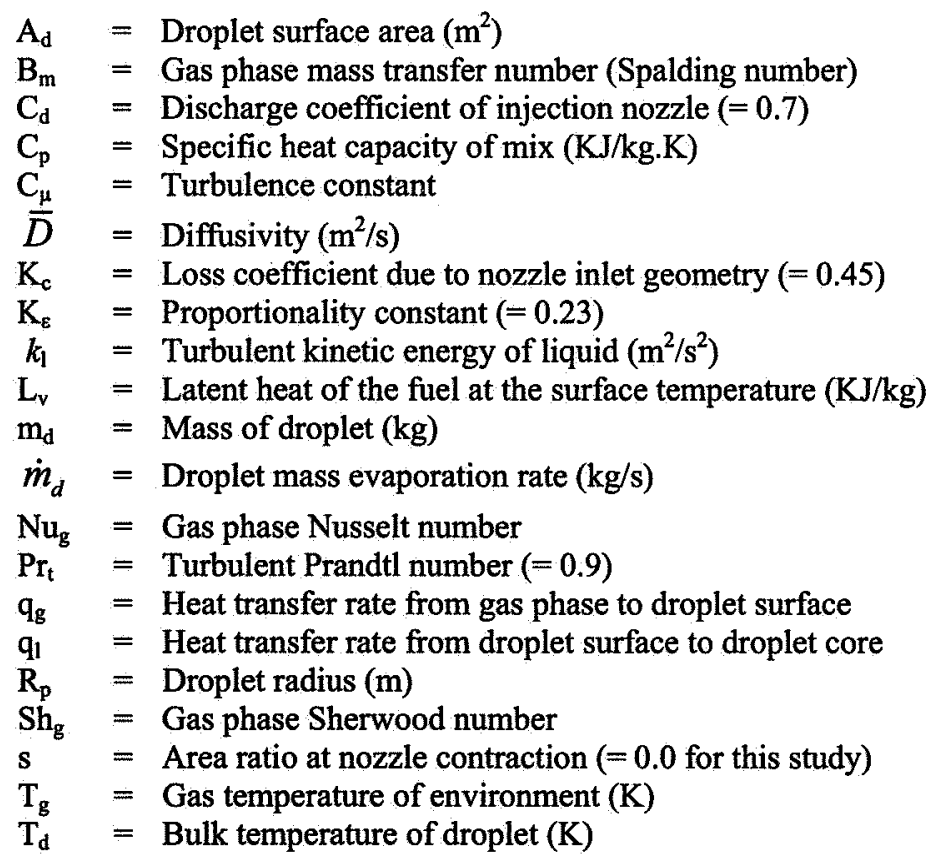

"Graduate Research Assistant, Student Member, AIAA.

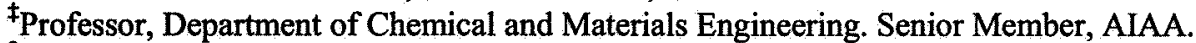

${ }^{\S}$ Aerospace Engineer, ER32/Combustion Devices Branch, NASA-Marshall Space Flight Center. 


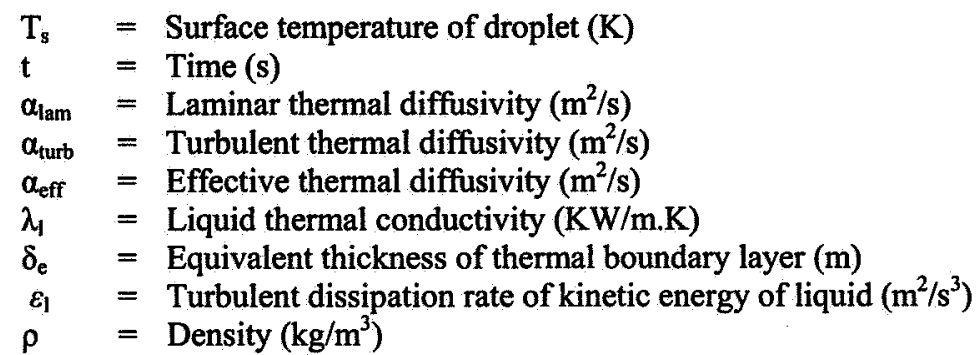

\section{INTRODUCTION}

Tn liquid fueled combustion systems such as rocket engines and ramjet/scramjet engine applications, the 1 combustion performance depends strongly on liquid atomization, spray vaporization, and the mixing process between the free stream environment and the liquid fuel spray. Smaller drop sizes generated from the spray devices increase the specific heat and mass transfer area of the fuel and thereby achieve high rates of mixing and evaporation. Consequently, higher engine efficiency would be realized in the combustion process. A large diversity of multi-phase gas-liquid flows of practical interest involves the evaporation of near spherical liquid droplets in high temperature turbulent environments. Such flows cover a wide range of applications including spray combustion. These situations involve a dispersed liquid phase species in the form of a large number of discrete droplets convecting and vaporizing in a continuous gas phase species. As droplets enter the hot free stream, only a small fraction of the liquid droplet near the liquid/gas interface heats up while the core region remains cold. The heat transfer through conduction and convection (possibly by internal turbulence and circulation) to its interior proceeds until the end of its lifetime. Resolving the transient phenomena within liquid droplets, as well as the interfacial heat/mass transfer are the subjects of various modeling approaches ${ }^{3-13}$. These evaporation models, when implemented in modern computational analyses utilizing the Eulerian-Lagrangian method, would involve calculations of velocity, temperature for both and vapor of the surrounding gases and the liquid droplets, as well as species mass fractions within the gas phase.

Most of the current droplet evaporation models used for computational analysis of spray combustion phenomena is based on the classical constant droplet-temperature model $\left(\mathrm{D}^{2}\right.$ law) or infinite conductivity (perfect mixing within the liquid droplets) models. Even the performance of the gas-side vaporization models may vary, droplet evaporation rates predicted by liquid-side infinite conductivity models tends to over predict or under predict the evaporation mass flux, depending on the ambient temperature conditions. Bertoli and Migliaccio ${ }^{3}$ showed that the accuracy of CFD computations of heating, evaporation and combustion of diesel fuel sprays could be substantially increased if the assumption of infinitely high thermal conductivity of liquid is relaxed. Sprays exposed to convective flows behave very differently from quiescent clouds of drops. Several modeling approaches have been proposed to account for finite conductivity effects within the evaporating droplets. Based on single drop analysis, Sirignano and Abramzon ${ }^{4-5}$ takes into account details of external flow around a droplet and the coupled internal flow dynamics inside the droplet for a droplet immersed in a convective flow. However these results are not applicable for dense spray conditions. Sazhin et al. ${ }^{6-7}$ pointed out that it is not economical (for CFD spray combustion) to resolve temperature profiles within evaporating droplets and proposed an alternative to numerically modeling the fuel droplet heating by obtaining analytical solutions to the heat conduction equation within the droplet. Miller et al. ${ }^{9}$ introduced non-equilibrium Langmuir-Knudsen evaporation law for use in droplet combustion models and found non-equilibrium effects to be important for droplet sizes found in practical spray calculations. Renksizbulut et al. ${ }^{10}$ developed a Nusselt number vaporization model which incorporated heat transfer enhancement due to internal circulation. Reveillion and Vervisch ${ }^{10}$ have considered the effects of both internal circulation and mixing inside the droplet. Takahashi et al. " modeled a turbulent thermal conductivity for the heat transfer behavior in the sheet region based on the model developed by Celata et al. ${ }^{12}$

The goal of this study is to extend a recently developed atomization/spray model ${ }^{1-2}$, the T-blob/T-TAB model, to include spray evaporation effects. Due to the unique feature of T-blob/T-TAB, in which the turbulence characteristics is accounted for within the droplet phase, extension of this model to include finite conductivity effect in the evaporating droplet can be made naturally. The model development will be described in this paper. Preliminary validations for one-way coupling, two-way coupling 2-D axis-symmetric and 3-D evaporating sprays will be presented with a long term goal of developing numerical capabilities for both low speed and high speed spray combustion applications including liquid turbulence effects. 


\section{NUMERICAL MODELING APPROACHES}

\subsection{T-blob and T-TAB Atomization/Spray Models}

The current vaporization model is developed for computational analysis based on the Eulerian-Lagrangian numerical approach. In this formulation, the spray/droplets dynamics is described in a Lagrangian coordinate such that numerical droplets are tracked within the Eulerian gas dynamics. Liquid phase is tracked from the injector plane, and the primary atomization, as well as the subsequent secondary breakup is modeled using the T-blob/TTAB hybrid model. Both primary and secondary droplet breakup processes are modeled and the transition from primary to secondary breakup is modeled based on energy balance. In addition to the droplets position and velocity, liquid turbulence is accounted for through the injector characteristics by the two-equation $k-\varepsilon$ turbulence model formulation using the T-blob/T-TAB model. Detailed model description and validations can be found in references 1 and 2, and it is suffice to say that within each numerical droplet, turbulence characteristics such as fluctuating velocity level, length and time scales are supplied by the model.

\subsection{The Finite Conductivity Evaporation Model}

To relax perfect mixing assumption within the liquid droplet, a "two-temperature model" is formulated. This type of two-temperature modeling has been described in the non-equilibrium evaporation models by Miller et al. ${ }^{8}$, and was used by $\mathrm{Ra}$ and Reitz ${ }^{13}$ for gasoline engine applications. In the two-temperature model, the core (or bulk) temperature $\left(T_{d}\right)$, is assumed well-mixed by convection/turbulence transport. In consistence with the "film theory", heat resistance exists at the near surface region, and the droplet surface temperature $\left(\mathrm{T}_{\mathrm{s}}\right)$ differs from the droplet core temperature. The heat transfer coefficient across this thin film (or boundary layer) is then formulated through the turbulence characteristics supplied from the T-blob/T-TAB model, to account for the finite conductivity effect. In the Lagrangian coordinate, the heat-up of the droplet core is formulated as:

$$
m_{d} C_{p} \frac{d T_{d}}{d t}=h_{l}\left(T_{s}-T_{d}\right) A_{d}
$$

where $h_{l}$ is the liquid-side heat transfer coefficient and $A_{d}$ is the droplet surface area. The heat transfer coefficient is determined from an effective thermal diffusivity formulation ${ }^{5}$ as:

$$
h_{l}=\frac{\lambda_{l}}{\delta_{e}} \text {, }
$$

where $\lambda_{l}$ is the liquid thermal conductivity and the film thickness, $\delta_{e}$, is given by $\sqrt{\pi \alpha_{\text {eff }} t}$. The effective thermal diffusivity $\left(\alpha_{e f f}\right)$, based on the turbulence characteristics within the droplet, is estimated from: $\alpha_{e f f}=\alpha_{\text {lan }}+\alpha_{t u r b}$, in which the turbulent thermal diffusivity is calculated from the two-equation turbulence model diffusivity formulation: $\alpha_{t u r b}=\frac{C_{\mu}}{\operatorname{Pr}_{t}} \frac{k_{l}^{2}}{\varepsilon_{l}}$, here $\operatorname{Pr}_{t}$ is the turbulent Prandtl number. The liquid droplet turbulence quantities $k_{l}$ and $\varepsilon_{l}$ are obtained from the T-blob/T-TAB atomization/spray model.

In cases where gas phase diffusivities are much larger than liquid diffusivities, the droplet core heating will be rate controlling and the gas-side heat/mass transfer will respond in a quasi-steady manner. The surface temperature of the droplet is determined from a heat and mass transfer balance at the interface between the droplet and the surrounding gas assuming no heat accumulation at the droplet surface such that:

$$
L_{v} \dot{m}_{d}=q_{g}-q_{l},
$$

where $L_{v}=$ latent heat of the fuel at the surface temperature $(\mathrm{KJ} / \mathrm{kg})$,

$q_{g}=$ heat transfer rate from the environmental gas to the surface. In this paper, the classical Spalding evaporation model is used to model the gas-phase transport, thus the gas heat transfer rate was calculated as: 


$$
=2 \pi D_{p} \lambda_{g} N u_{g}\left(T_{g}-T_{s}\right) \frac{\ln (1+B)}{B}
$$

$q_{1}=$ heat transfer rate from the droplet interior to droplet surface $=\frac{\lambda_{1}\left(T_{s}-T_{d}\right)}{\delta_{e}} A_{s}$, and the evaporation rate at the surface is given as:

$$
\dot{m}_{d}=\frac{d m_{d}}{d t}=2 \pi D_{p}\left(\rho_{g} \bar{D}\right) S h_{g} \ln \left(1+B_{m}\right)
$$

In the above equation, $B_{m}$ is the Spalding mass transfer number, $S h_{g}$ is the Sherwood number, and $\bar{D}$ is the binary diffusivity. The Sherwood and the Nusselt numbers were calculated using the classical correlations, given by:

$$
\begin{aligned}
& N u_{g}=1+0.3 \operatorname{Re}_{p}{ }^{0.5} \operatorname{Pr}^{0.333} \\
& S h_{g}=1+0.3 \operatorname{Re}_{p}^{0.5} S c^{0.333}
\end{aligned}
$$

The equilibrium condition at the droplet surface is determined by the Clausius-Clayperon relationship.

\section{VALIDATION RESULTS AND DISCUSSION}

Several test cases were selected to investigate the performance of the current two-temperature evaporation model. Initially, a simple FORTRAN program was written for one-way coupled evaporating atomizing spray in a quiescent gas. This program is an extension of the T-blob/T-TAB code written by Trinh and Chen ${ }^{1}$. Detailed numerical calculation issues such as time step, initial conditions and the iterative loop of obtaining droplet surface temperature and core temperatures can be found in Balasubramanyam ${ }^{14}$. After the initial testing, the current model then was implemented into an existing CFD code, CFD-ACE ${ }^{15}$, for two-way coupling Eulerian-Lagrangian calculations. Both 2-D axis-symmetric co-flowing cases and 3-D cross flow cases were investigated. Again, Ref. 14 should be consulted for detailed numerical (such as grids and time steps) implementation issues.

\subsection{One-Way Coupled Evaporating Atomizing Spray}

This case is an extension of the one-way T-blob/T-TAB testing case described in Trinh and Chen ${ }^{1}$. The purpose here is to investigate the concept of boundary layer film thickness within with liquid droplets involving two temperatures. In the course of study, it was found that, due to the fact, in the secondary breakup regime (i.e. the TTAB regime) the droplets were so small they were heated up rapidly. Thus the current model was only implemented within the T-blob (i.e., the primary breakup) model. The Tridecane fuel was issued through a long injector tube at $300^{\circ} \mathrm{K}$. The length of the injector nozzle (L) is $0.8 \mathrm{~mm}$ and the nozzle diameter $\left(D_{n o z z l e}\right.$ ) is $0.3 \mathrm{~mm}$. A jet injection velocity (U) of $102 \mathrm{~m} / \mathrm{s}$ was used for one of the test cases. Based on these values, the initial conditions for liquid turbulent kinetic energy and its dissipation rate are estimated ${ }^{1}$ as:

$$
\begin{aligned}
\mathrm{k}_{\mathrm{l}}^{\mathrm{o}} & =\frac{\mathrm{U}^{2}}{8 \mathrm{~L} / \mathrm{D}_{\text {nozale }}}\left[\frac{1}{\mathrm{C}_{\mathrm{d}}^{2}}-\mathrm{K}_{\mathrm{c}}-\left(1-\mathrm{s}^{2}\right)\right] \\
\varepsilon_{1}^{0} & =\mathrm{K}_{\varepsilon} \frac{\mathrm{U}^{3}}{2 \mathrm{~L}}\left[\frac{1}{\mathrm{C}_{\mathrm{d}}^{2}}-\mathrm{K}_{\mathrm{c}}-\left(1-\mathrm{s}^{2}\right)\right]
\end{aligned}
$$

The environment is quiescent nitrogen at a temperature of $600^{\circ} \mathrm{K}$. The gas properties were calculated based on the reference state determined from the ' $1 / 3^{\text {rd }}$ ' rule. $^{5}$. In this calculation, a 'blob' of numerical droplet was injected at the orifice plane with orifice diameter. The droplet then went through first and secondary breakup processes, thus its diameter decreased in time. The variation of the thermal boundary layer within the droplet also changed in time. In figure 1, the change of the thermal boundary layer normalized with the droplet radius versus time is shown for the test case of a droplet evaporating in a quiescent environment. It was also observed that the turbulent diffusivity evaluated using the dispersed phase flow turbulence was at least two orders of magnitude higher than the laminar thermal diffusivity. The result shows that the normalized thermal boundary layer is rather small and exhibits reasonable physical trend. Detailed droplet characteristic history, such as the two temperature history, heat up time, 
as well as turbulence characteristics can be found in Ref. 14. The model was then incorporated into the finite volume commercial CFD code ACE $+{ }^{15}$. Current work is focused towards validation of the code with test cases for which experimentally measured data is available for comparison. Some of these results will be presented in this paper.

\subsection{Two-Way Coupled Evaporating Spray Validation}

To evaluate the current evaporation model, the 2-D axis-symmetric subsonic low-speed evaporating spray of Yakota et al. ${ }^{16}$ was tested first for a two-way coupling CFD calculation since a similar non-evaporating was used for the T-blob/T-TAB validation study ${ }^{2}$. Liquid fuel (Tridecane $\mathrm{C}_{13} \mathrm{H}_{28}$ ) is injected through a single-hole nozzle into a high pressure, high temperature ambient $\mathrm{N}_{2}$ environment. The initial test conditions for the evaporating spray are summarized in Table 1.

Table 1. Test Conditions for the Measurement of Yokota et al. ${ }^{16}$

\begin{tabular}{cccccc}
\hline Case & $\mathrm{P}_{\mathrm{inj}}$ & $\mathrm{P}_{\mathrm{gas}}$ & $\mathrm{T}_{\text {amb }}$ & $\mathrm{M}_{\mathrm{inj}}$ & Gas Environment \\
\hline $\mathrm{Y}-1$ & $30 \mathrm{Mpa}$ & $3 \mathrm{Mpa}$ & $900 \mathrm{~K}$ & $0.00326 \mathrm{~kg} / \mathrm{s}$ & $\mathrm{N}_{2}$ \\
\hline
\end{tabular}

The nozzle diameter was $0.16 \mathrm{~mm}$. Equations (7) and (8), with the same values for nozzle parameters except for nozzle diameter and jet velocity, were used to estimate the initial liquid turbulence quantities. A computational domain of $20 \mathrm{~mm}$ in radius and $100 \mathrm{~mm}$ in length discretized by a 50 radial x 75 axial grid was used. The mesh spacing was non-uniform with refinement on the centerline and close to the injector. A constant time step of 2.5E-6 sec. was used with an injection period of $4 \mu \mathrm{sec}$. The properties of liquid fuel Tridecane were taken from the NIST/JANAF database. Estimating the penetration of a fuel jet into an air stream is an important global property for model validation and is presented first. In Figure 2, the predicted tip penetration results using the current finiteconductivity (F-C) evaporation model coupled with the T-blob/T-TAB atomization model are compared with the measured data. For reference, predictions using classical Blob/TAB/infinite-conductivity (I-C) model, as well as using $\mathrm{T}-\mathrm{blob} / \mathrm{T}-\mathrm{TAB} /$ infinite-conductivity model are also shown in the same figure. It can be seen that the infiniteconductivity evaporation models tend to over predict the evaporation rate, thus gave shorter tip penetration. On the other hand, the finite-conductivity model slows down the evaporation process, and produce larger droplets and longer penetration. In figure 3.b, it can be observed that larger numerical droplets are clustered near the tip region due to slower evaporation process calculated by the finite-conductivity model, when compared with the parcel distribution using infinite-conductivity model 3.a, $1 \mu \mathrm{sec}$ after the injection. It should be noted that the coalescence model $^{2}$ was used for all two-way coupling calculation cases. The coalescence model is responsible for the calculated overshoot phenomena observed in the initial period of injection for all simulated cases.

The heat and mass transfer aspects of the evaporating jet are shown in figures 4 and 5 at time of $4 \mu \mathrm{sec}$ after injection. As can be observed, the models incorporating the turbulence effects in the primary and secondary atomization processes give more reasonable qualitative pictures when compared with the classical atomization model without liquid turbulence effect. Comparing figures $4 . b$ and 4.c, the surface temperature contours predicted by the current finite-conductivity model show the effect of slowing down the rate of evaporation, based on the turbulence levels experienced by the individual droplets, rather than the constant drop temperature assumption of the classical infinite conductivity model. The fuel mass fraction contours for test case $\mathrm{Y}-1$ are as indicated in figures 5.a, 5.b and 5.c. The comparisons between figures 5.b and 5.c show that diffusion of the fuel species into the environment is at much lower rate than the case with the classical infinite conductivity model.

\subsection{Two-Way Coupled 3-D Supersonic Cross-Flow Validation}

The study of liquid atomization process in a high-speed flow has become an important area of research in airbreathing vehicle (Scramjet) development. The breakup process in a cross-flow involve the effects of drop deformation, displacement, breakup time and ignition were stidied by Ranger and Nichols ${ }^{19}$ and Shetz et al. ${ }^{20}$. Though advances (Gruenig and Mayinger ${ }^{21}$, Yu et al. ${ }^{22}$, Vinogradov et al. ${ }^{23}$, Inamura et al. ${ }^{17}$ ) in this area of research have been made it is still not progressed so far as to provide validation cases for evaporation measurements due to the complexity of the phenomena occurring in these types of situations and the lack of measurement techniques to 
capture these phenomena. Owing to limitations in availability of data for comparisons it was decided to apply the Tblob/T-TAB (originally developed for low speed parallel injector flows) model coupled with the infinite conductivity model, for simulating the test case of Lin et al. ${ }^{24}$ (also used by $\mathrm{Im}$ et al. ${ }^{18}$ ) for the injection of a water jet into a cross-flow at Mach 1.94.

A three dimensional computational domain with dimensions of $762 \times 152 \times 127 \mathrm{~mm}$, discretized with a $90 \times 25 \times 30$ grid distribution, with refinement at the injection point was used for the numerical computations. The inlet boundary conditions for the chamber were given by a static pressure of $29 \mathrm{KPa}$, a static temperature of $304.1^{\circ} \mathrm{K}$ and a flow velocity of $679 \mathrm{~m} / \mathrm{s}$ based on a free stream Mach number of 1.94 . The injection velocity of the spray jet was determined based on a jet-to-air momentum ratio $\mathrm{q}_{0}=7.0$. In this study the water jet injection temperature was set to $300^{\circ} \mathrm{K}$. The injection nozzle diameter was $0.5 \mathrm{~mm}$ and located at a distance of $139 \mathrm{~mm}$ from the chamber inlet. Again, inlet turbulence quantities are estimated by Eqs. (7) and (8) due to the lack of information. A computational time step of $2.5 \mathrm{E}-6 \mu \mathrm{sec}$ was used with an injection period of $4 \mu \mathrm{sec}$.

The cross-section of the jets is circular at the injector exit and becomes kidney shaped due to the dynamic pressure of the air stream. This phenomenon was observed by Shetz and Thomas ${ }^{25}$. The spray tip penetration for this cross-flow test case is shown in figure 6 . The jet penetration increased rapidly in the vicinity of the injector exit and then gradually increased due to increase in the drag of the air stream. The momentum of the spray droplets increases due to acceleration by the airflow in the direction of the supersonic airflow. Consequently, the penetration curve of the spray plume approaches the airflow direction as observed in figure 7. Figures 8 and 9 show the Mach and pressure contours at the mid-plane cut of the evaporating spray. An experimental correlation curve suggested by Lin et al. ${ }^{24}$ is also shown in figure 6 . It should be pointed out that the current simulation employs the same T-blob/TTAB model and the associated low-speed droplet drag law without adjusting any model constants. However, the droplets have been observed to accelerate to supersonic speeds at a later part of the spray. The evaporation of droplets would also result in a lowering of the drag force of the air stream ${ }^{17}$. Further improvement in drag law to account for high speed effect is definitely needed. In addition, the inlets liquid jet turbulence level was found to be quite sensitive in terms of cross-flow spray penetration calculation ${ }^{14}$. Recently, inlet liquid jet turbulence is also observed to be sensitive to spray dynamics experimentally ${ }^{21}$. Considering the uncertainties associated with the jet inlet conditions, the comparison with experimental data is reasonable. Further numerical studies should be conducted to clarify the effects of air temperature on jet penetration incorporating the finite-conductivity evaporation model.

\section{CONCLUDING REMARKS}

We have developed a new finite-conductivity model, based on the two-temperature film theory, for evaporating spray numerical calculations. The model is a natural extension of the T-blob/T-TAB atomization/spray model which supplies the turbulence characteristics for estimating effective thermal diffusivity within the droplet. Based on oneway coupling simple spray results, the model exhibits reasonable physical trends in terms of droplets evaporation features. In two-way Eulerian-Lagrangian multi-dimensional full CFD simulations, the current finite-conductivity model coupled with the T-blob/T-TAB model show superior performance to conventional infinite-conductivity evaporation models when comparing to low-speed parallel injector flow experimental data. Due to the lack of high speed evaporating spray data, limited results using the same T-blob/T-TAB model for high speed cross flow spray were presented. Extension of the low-speed T-blob/T-TAB model for high-speed cross flow application show surprisingly reasonable results considering the uncertainties involved. The preliminary high speed evaporating spray study also pointed to the future directions of improving the current model in terms of high speed droplet drag laws, additional breakup mechanisms, and needs of detailed quality experimental data.

\section{ACKNOWLEGEMENTS}

This study was partially supported by NASA Grant NCC8-200 through the UAH Propulsion Research Center. Technical consultations of Dr. Ashok Gidwani and Dr. Kapil Pant, of CFD Research Corporation, in this study are acknowledged. The authors would also like to acknowledge the use of the CFD-ACE+ code under an educational agreement with CFD Research Corporation. 


\section{REFERENCES}

'Trinh, H. P., and Chen, C. P., "Modeling of Turbulence Effects on Liquid Jet Atomization \& Breakup", to appear in Atomization and Spray, also AIAA-2005-0154, 2005.

${ }^{2}$ Trinh, H. P., and Chen, C. P., "Numerical Simulation of Liquid Jet Atomization Including Turbulence Effects", AIAA-2005-3973, 2005.

${ }^{3}$ Bertoli, C., and Migliaccio, M. na., "A Finite Conductivity Model for Diesel Spray Evaporation Computations", Int. Journal of Heat \& Fluid Flow, Vol. 20, pp. 552-561, 1999.

"Abramzon, B., and Sirignano, W. A., "Droplet Vaporization Model for Spray Combustion Calculations", Int. J. Heat and Mass Transfer, Vol. 32, No. 9, pp. 1605-1618, 1989.

${ }^{5}$ Sirignano, W. A., "Fluid Dynamics and Transport of Droplets and Sprays", Cambridge University Press, 1999.

${ }^{6}$ Sazhin, S. S., Abdelghaffar, W. A., Krutitskii, P. A., Sazhina, E. M., and Heikal, M. R., "New Approaches to Numerical Modeling of Droplet Transient Heating and Evaporation", Int.Journal of Heat and Mass Transfer, Vol. 48, No. 19-20, pp. 4215-4228, 2005.

${ }^{7}$ Sazhin, S. S., Krutitskii, P. A., Abdelghaffar, W. A., Sazhina, E. M., Mikhalovsky, S. V., Meikle, S. T., and Heikal, M. R.," "Transient Heating of Diesel Fuel Droplets", Int. Journal of Heat and Mass Transfer, Vol. 47, No. 14-16, pp. 3327-3340, 2004.

${ }^{8}$ Miller, R. S., Harstad, K., and Bellan, J., "Evaluation of Equilibrium and Non-Equilibrium Evaporation Models for Many-Droplet Gas-Liquid Flow Simulations", Int. Journal of Multiphase Flow, Vol. 24, pp. 1025-1055, 1998.

${ }^{9}$ Renksizbulut, M., Bussmann, M., and Xianguo, L., "A Droplet Vaporization Model for Spray Calculations", Particle and Particulate System Charaterization, Vol. 9, pp. 59-65, 1990.

${ }^{10}$ Reveillion, J., and Vervisch, L., "Spray Vaporization in Non Premixed Turbulent Combustion Modeling: A Single Droplet Model", Combustion \& Flame, Vol. 121, pp. 75-90, 2000.

"Takahashi, M., Nayak, A. K., Murakoso, H., and Kitagawa, S.,"Study on Vapor Condensation Heat Transfer to Liquid Spray", $7^{\text {th }}$ Int. Conf. on Nuclear Engineering, Tokyo, 1999.

${ }^{12}$ Celata, G. P., Cumo, M., D'annibale, F. and Farello, G. E., "Direct Contact Condensation of Steam on Droplets", Int. J. Multiphase Flow, Vol. 17, No. 2, pp. 191-211, 1991.

${ }^{13} \mathrm{Ra}$, Y., and Reitz, R. D., "The Application of a Multicomponent Droplet Vaporization Model to Gasoline Direct Injection Engines", Int. J. Engine Research, Vol. 4, No. 3, pp. 193-218, 2003.

${ }^{14}$ Balasubramanyam, M. S., "Modeling Turbulence Effects on the Heat and Mass Transfer of Evaporating Sprays", Ph.D. Dissertation, Department of Mechanical and Aerospace Engg., University of Alabama in Huntsville, 2006.

${ }^{15}$ CFD Research Corporation, "ACE+ Theory Manual", 2004.

${ }^{16}$ Yokota, H., Kamimoto, T., and Kobayashi, H., "A Study of Diesel Spray and Flame by an Image Processing Technique", Bulletin of JSME, Vol. 54, No. 71, 1988.

${ }^{17}$ Inamura, T., Takahashi, M., and Kimakawa, A., "Combustion Characteristics of a Liquid Fueled Ramjet Combustor", Journal of Propulsion and Power, Vol. 17, No. 4, pp. 860-868, 2001.

${ }^{18} \mathrm{Im}$, K. S., Lin, K. C., and Lai, M. C., "Spray Atomization Model of Liquid Jet in Supersonic Cross Flows", $43^{\text {rd }}$ Aerospace Sciences Meeting, Reno, AIAA 2005-0732, 2005.

${ }^{19}$ Ranger, A. A., and Nichols, J. A., "Aerodynamic Shattering of Liquid Drops", AlAA Journal, Vol. 7, No. 2, pp. 285-290, 1969.

${ }^{20}$ Schetz, J. A., Cannon, C., and Baranovsky, S., "Ignition of Liquid Fuel Jets in a Supersonic Air Stream", AIAA Journal, Vol. 18, No. 9, pp. 1101-1102, 1980.

${ }^{21}$ Mayinger, F., and Gruenig, C., "Supersonic Combustion of Kerosene/ $\mathrm{H}_{2}$ Mixtures in a Model Scramjet Combustor", Inst.for Thermodynamics, Munich, Germany, 2005.

${ }^{22}$ Yu, G., Li, J. G., Yue, J. R., Zhao, J. R., Zhang, X. Y., Huang, Y., and Sung, C. J., "Characterization of Kerosene Combustion in Supersonic Flow Using Effervescent Atomization", $11^{\text {th }}$ AIAA/AAAF Conference, AIAA 20025225.

${ }^{23}$ Vinogradov, V. A., Kobigsky, S. A., and Petrov, M. D., "Experimental Investigation of Kerosene Fuel Combustion in Supersonic Flow", Journal of Propulsion and Power, Vol. 11, No. 1, pp. 130-134, 1995.

${ }^{24}$ Lin, K.C., Kennedy, P. J., and Jackson, T. A., "Structures of Water Jets in a Mach 1.94 Supersonic Crossflow", 42nd AIAA Aerospace Sciences Meeting and Exhibit, AIAA Paper 2004-0971, Jan 2004.

${ }^{25}$ Shetz, J. A., and Thomas, R. H., "Distribution Across the Plume of Transverse Liquid and Slurry Jets in Supersonic Flow", AIAA Journal, Vol. 23, No. 12, pp. 1892-19่01, 1985. 


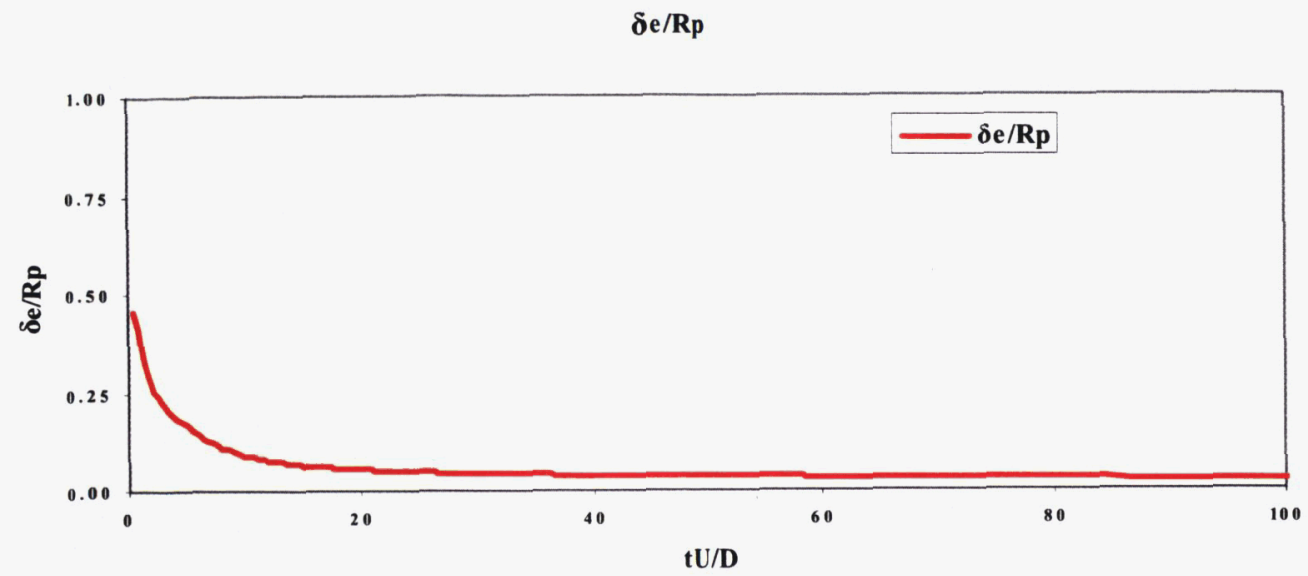

Figure 1. Variation of the normalized thermal boundary layer with time.

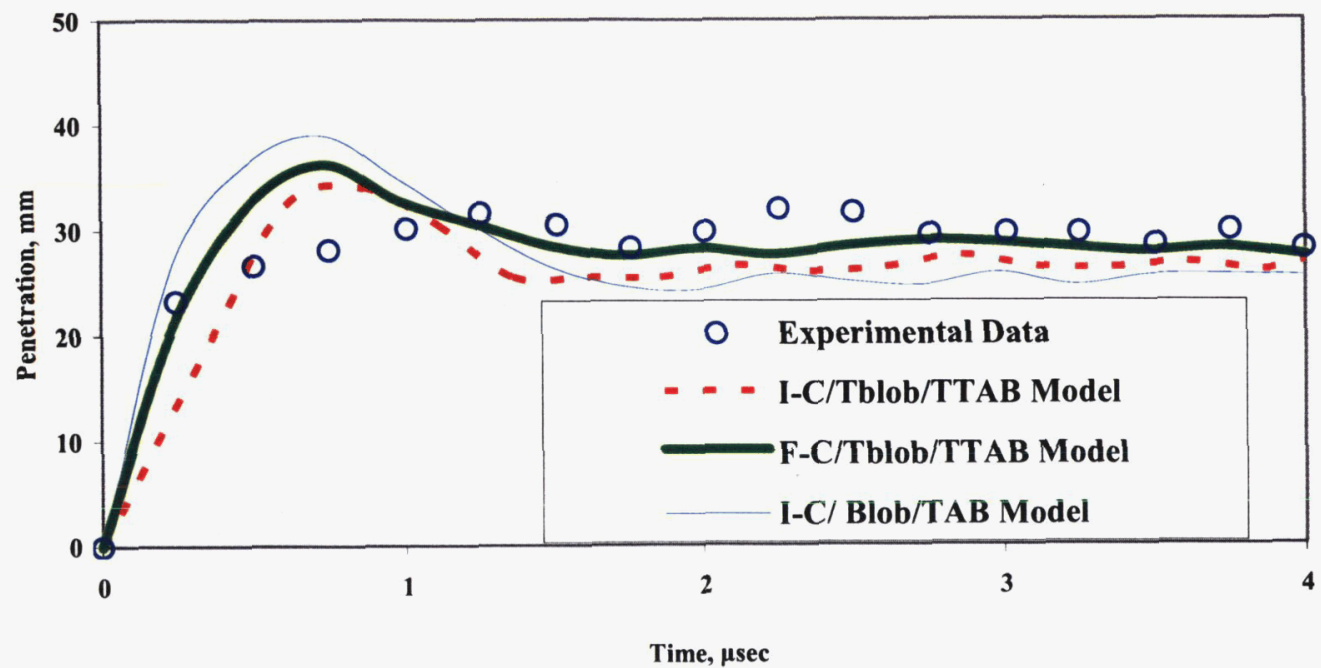

Figure 2. Spray Tip Penetration with time Comparisons

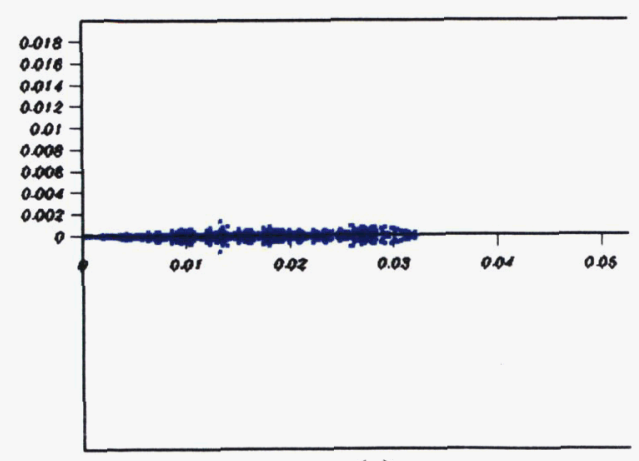

(a)

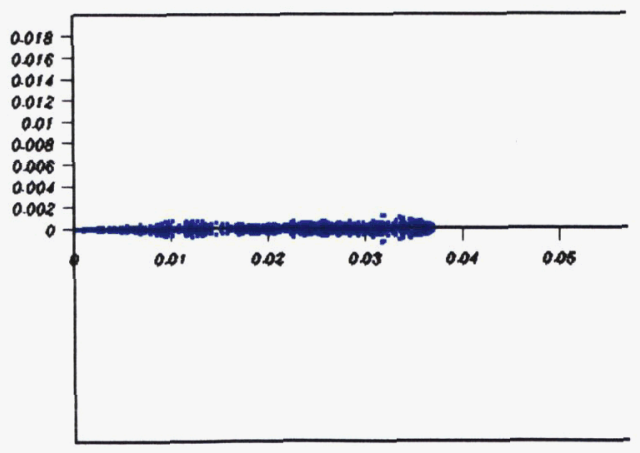

(b)

Figure 3. Spray Parcel Distribution with T-blob and TTAB at $1 \mu$ sec; (a) I-C Model (b) F-C Model 


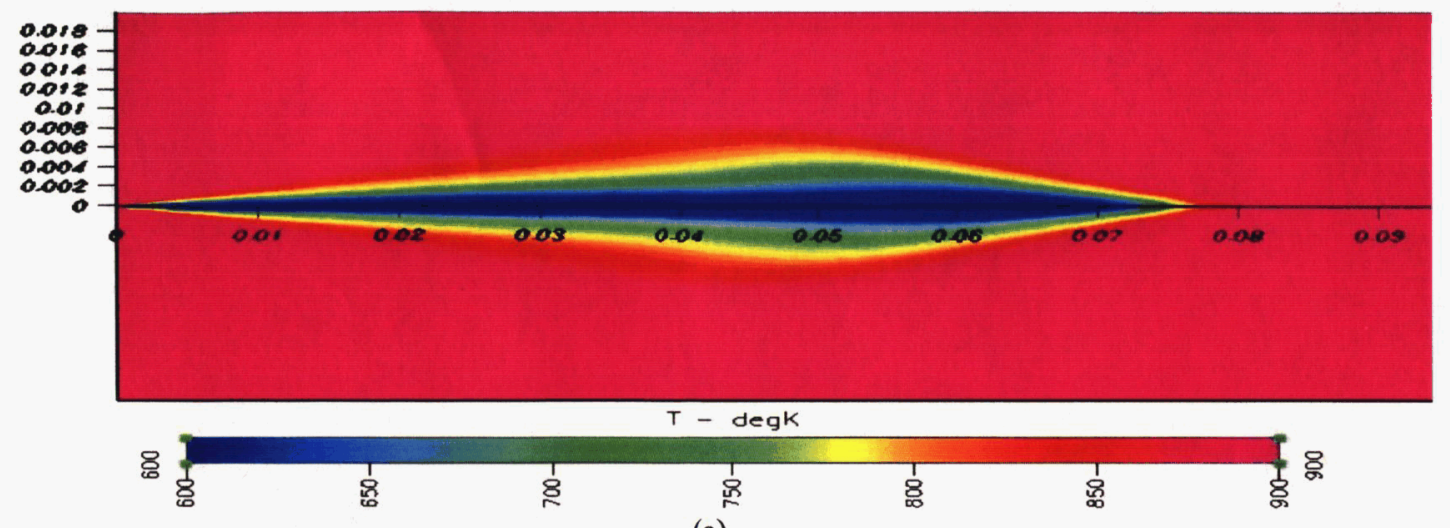

(a)

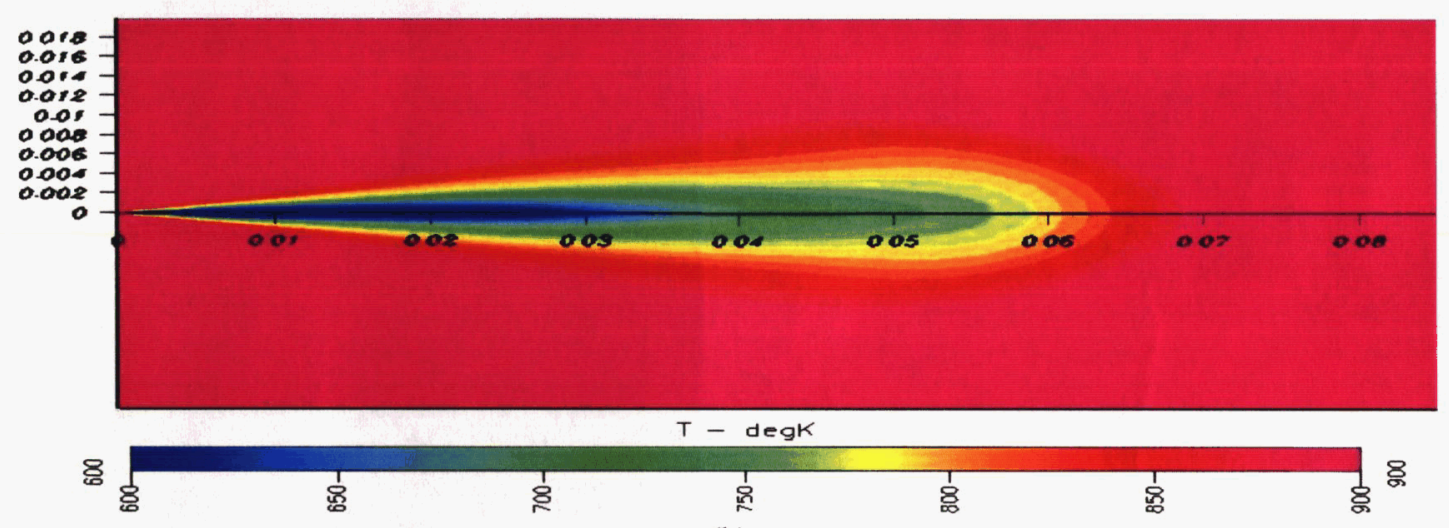

(b)

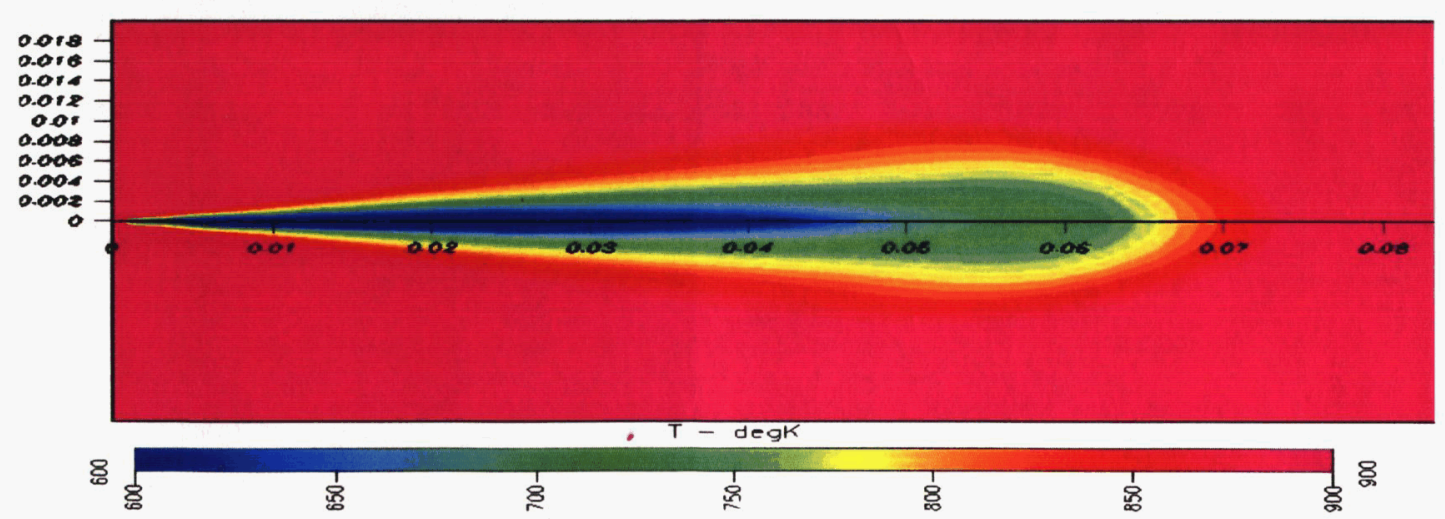

(c)

Figure 4. Temperature Contours at $4 \mu \mathrm{sec}$; (a) I-C with Blob/TAB Model, (b) I-C with T-blob /TTAB, (c) F-C with T-blob /TTAB 

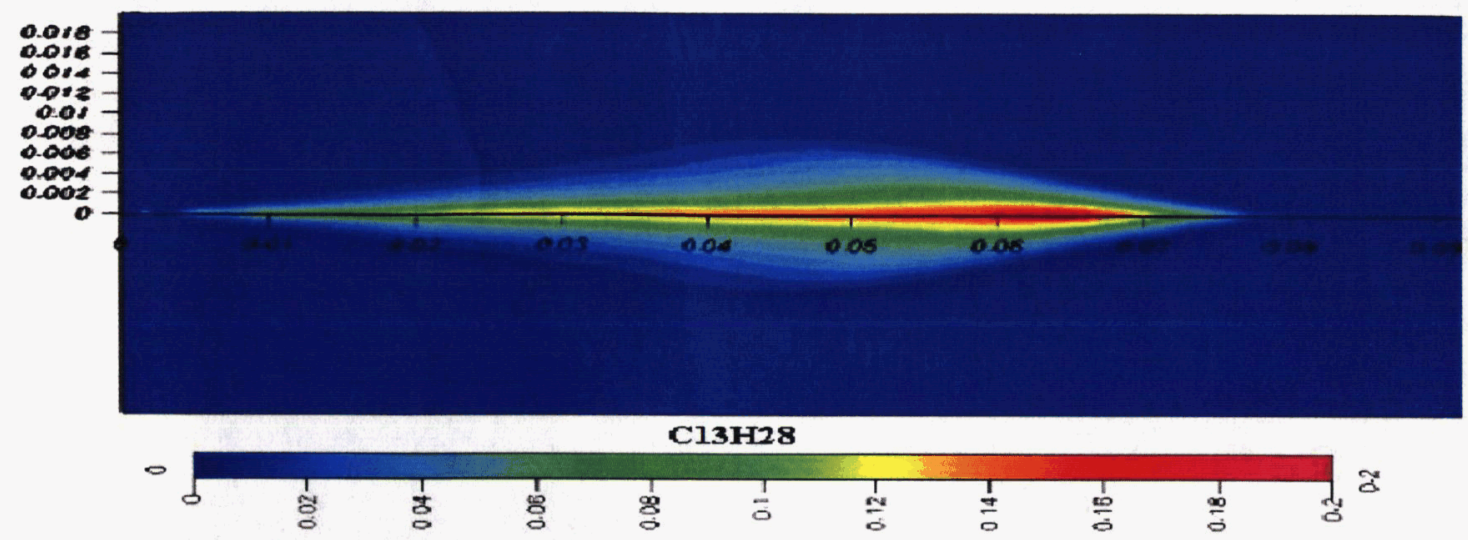

(a)

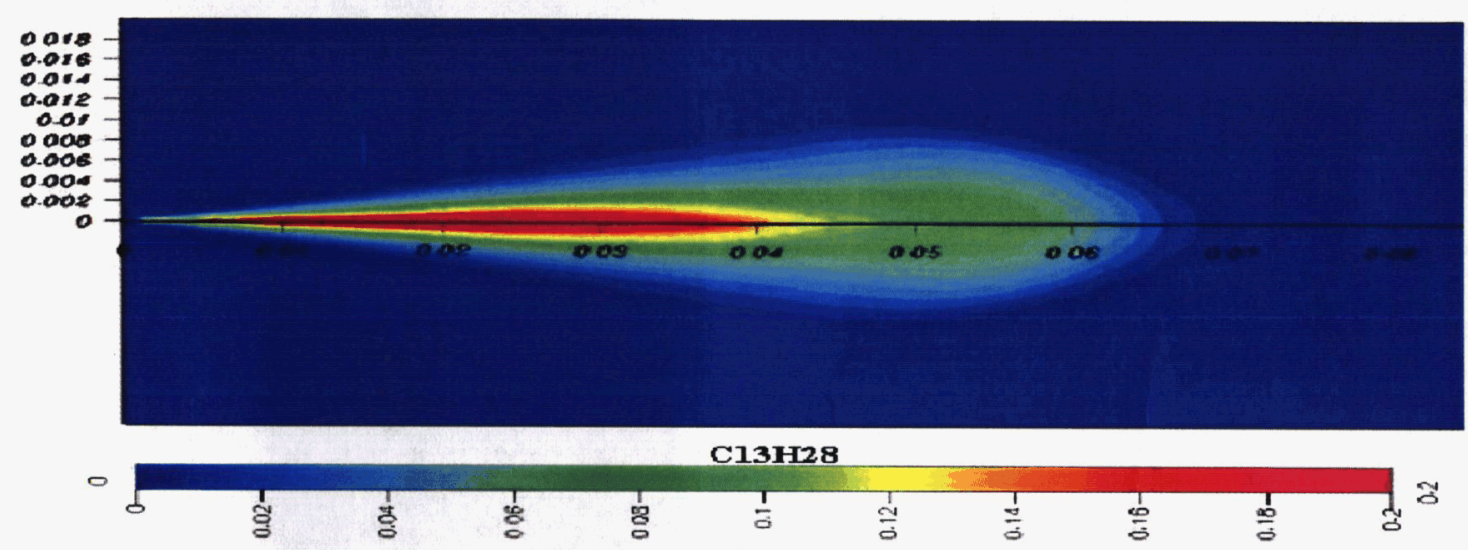

(b)

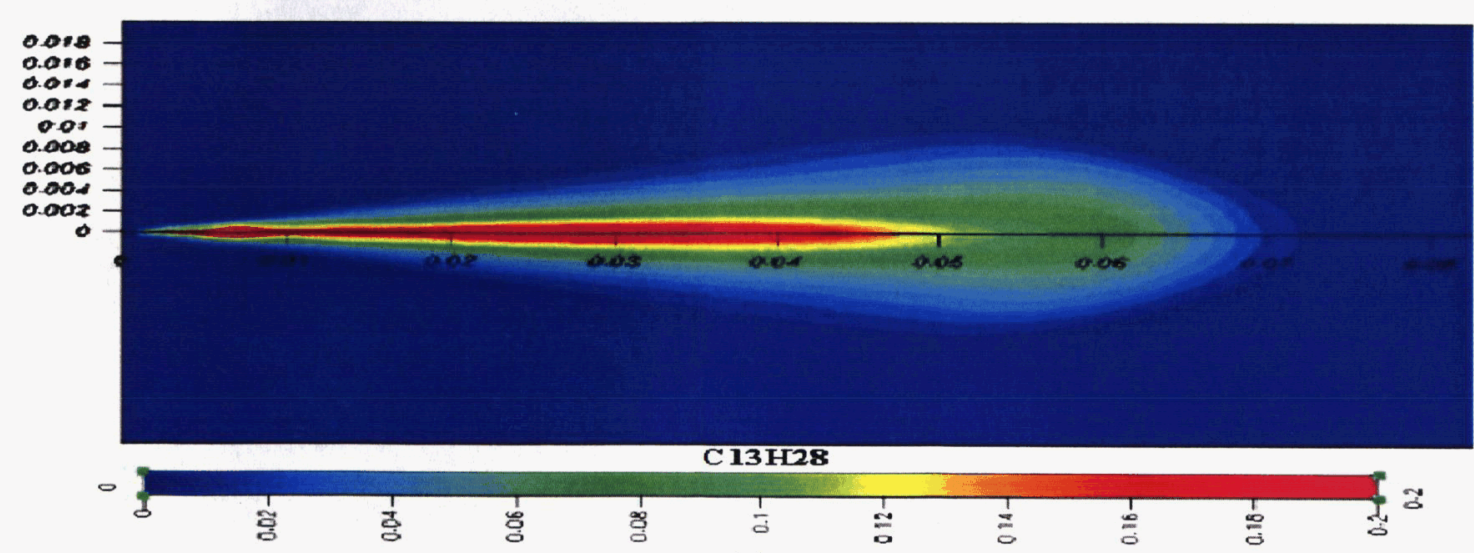

(c)

Figure 5. Fuel Mass Fraction Contours at $4 \mu \mathrm{sec}$; (a) I-C with Blob/TAB Model, (b) I-C with T-blob /TTAB, (c) F-C with T-blob/TTAB Model 


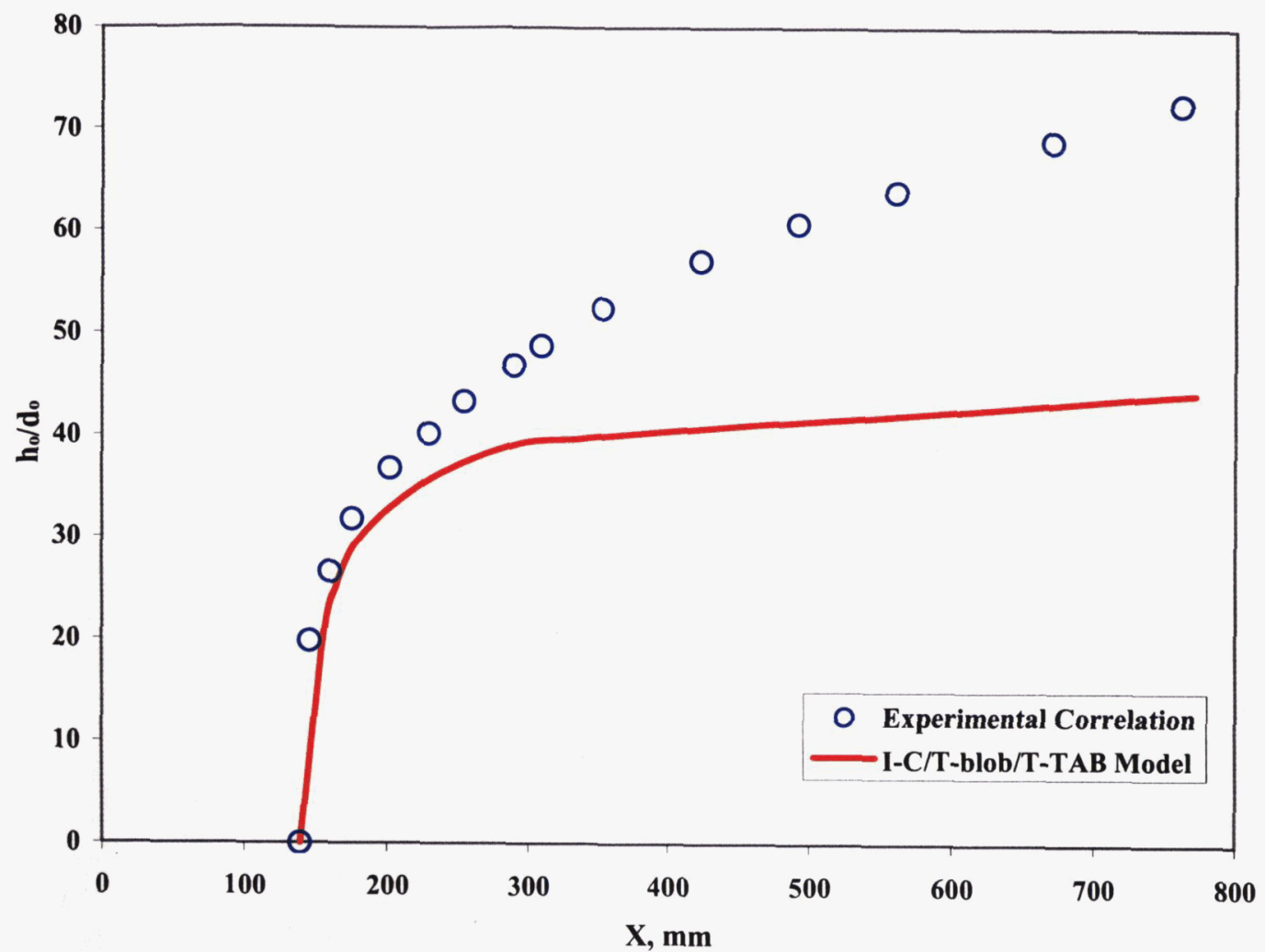

Figure 6. Spray Tip Penetration Comparisons

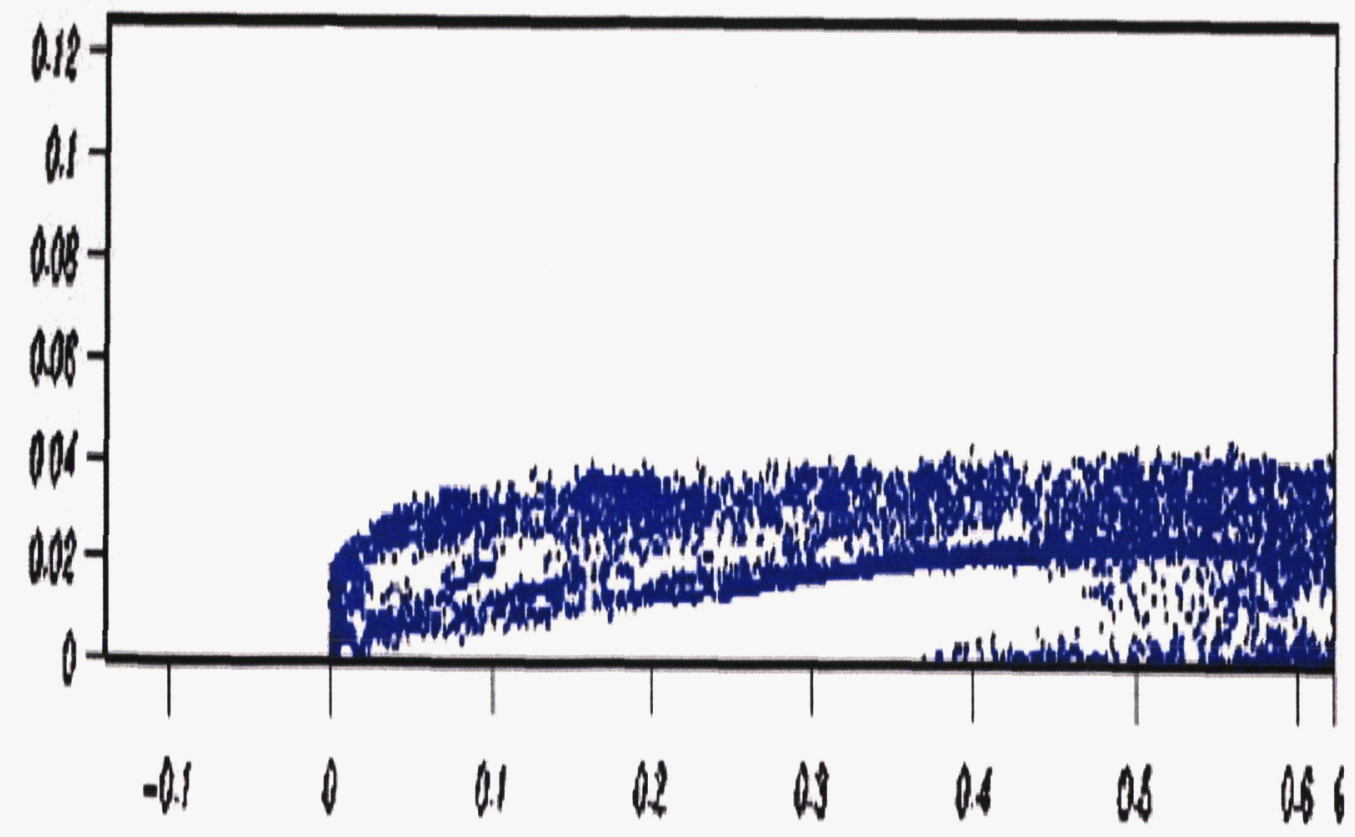

Figure 7. Jet penetration pattern at mid plane 

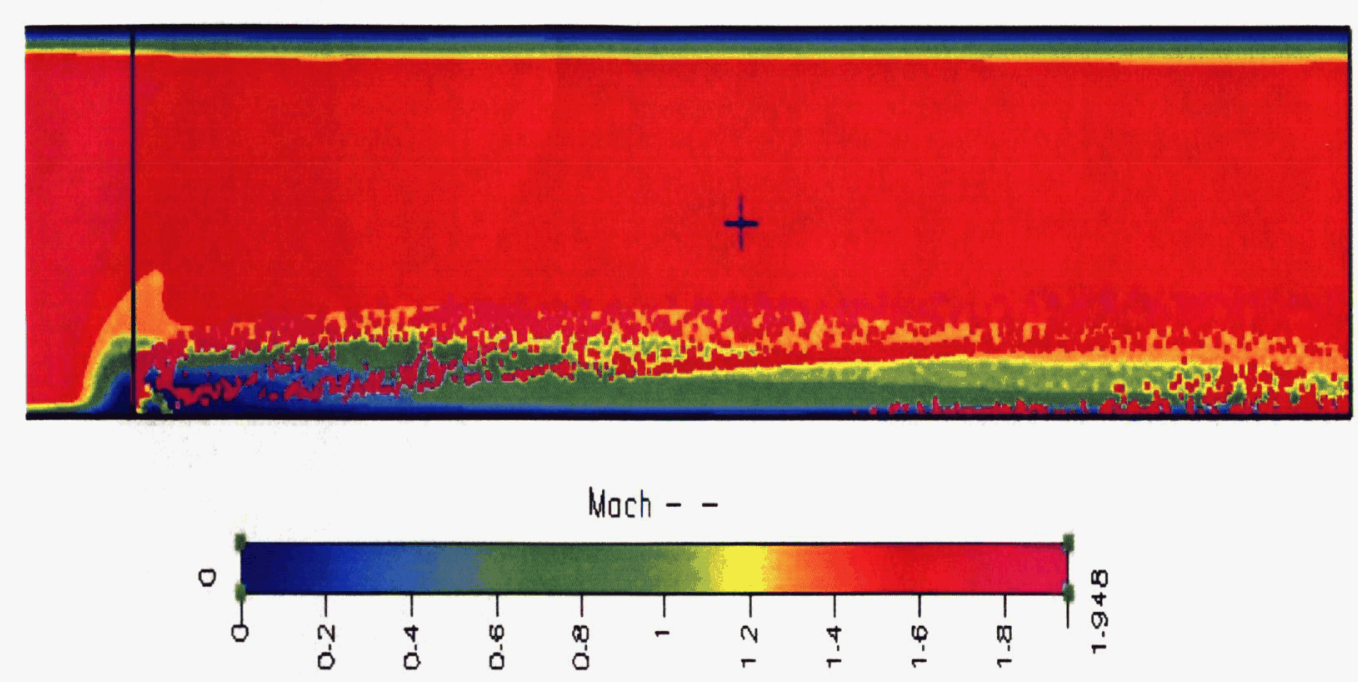

Figure 8. Mach number contours - I-C with T-Blob/T-TAB Model

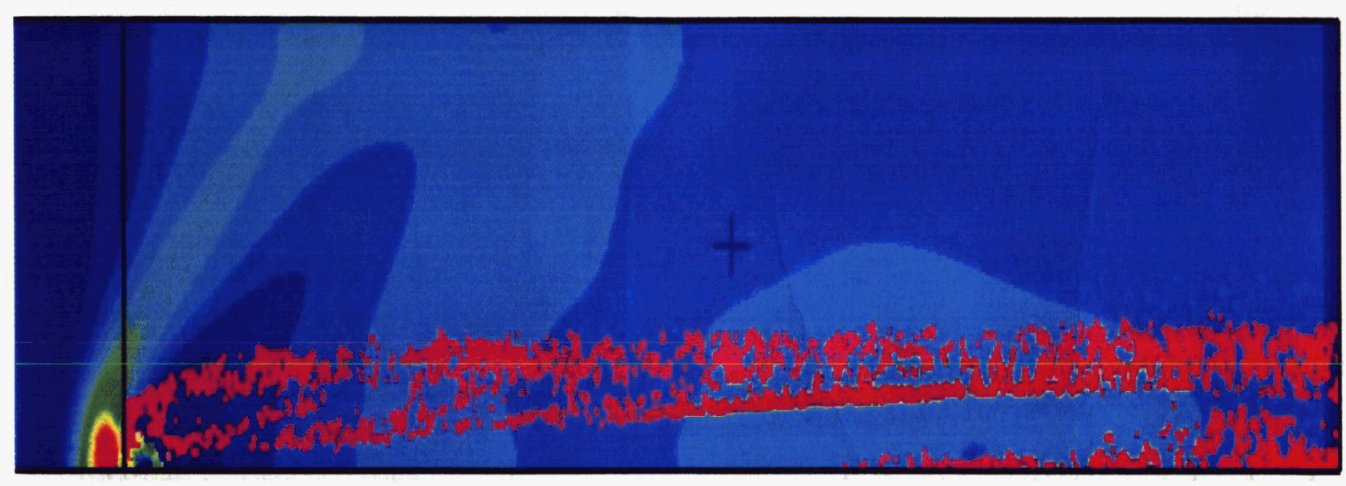

Figure 9. Pressure contours - I-C with T-Blob/T-TAB Model 